soluble products from other tumours become measurable.

At the end of the final session, Dr R. C Gallo (National Institutes of Health, Bethesda) summarized the current state of play with reverse transcriptase. Only one virus not known to be oncogenic has been found so far to possess the enzyme and he felt it could still be relevant to neoplasia even if not specific to tumour cells. $\mathrm{He}$ also brought news of a type $\mathrm{C}$ virus isolated from an American Burkitt lymphoma which is associated with RNA dependent DNA polymerase, and as a final titbit he reported that by screening more than a hundred rifamycin derivatives, a small number with high anti-polymerase activity had been obtained.

\section{MARS}

\section{Microwave Specirum}

from a Correspondent

THE spectrum of the microwave radiation from the planet Mars is a fascinating enigma which is still poorly understood. A controversy over the correct interpretation of the spectrum is exposed in the current issue of Icarus which carries several mutually exclusive research notes. The simplest prediction, based on the equation of heat conduction, is that the measured brightness temperature of the planet will show an increase at shorter wavelengths. This is because long waves record the mean planetary temperature, whereas shorter waves penetrate to shallower depths which are heated up by the solar radiation. In principle the location of the spectral turn up expected somewhere in the millimetre wave region gives information on the thermal and electrical properties of the surface, but, unfortunately, the observations will not fit this naive pattern.

In such a confused situation it is gratifying that several groups are striving to improve the quality of the data, particularly in the critical zone below 1 $\mathrm{cm}$ wavelength. Observations made during the 1969 opposition at the Crimean Astrophysical Observatory yield temperatures of $240 \mathrm{~K}$ at $2.3 \mathrm{~mm}$ and $210 \mathrm{~K}$ at $8.15 \mathrm{~mm}$. These values are considerably higher than those obtained in other experiments, and in fact V. A. Efanov et al. (Icarus, 14, 198 ; 1971) claim them as evidence for a temperature enhancement at short wavelengths. In order to do this, however, they have arbitrarily to discard some of the data.

Contrasting with this result, a similar measurement at $8.22 \mathrm{~mm}$ by A. D. Kuz'min et al. of Moscow (ibid., 192) gave a temperature of $176 \mathrm{~K}$ which is indicative of a turn down in the spectrum. When these authors attempted to relate other data to this point it became obvious from the many contra- dictory measurements that no simple conclusion is possible. Falling neatly between the sets of Russian measurements is a further result by $R$. W. Hobbs and S. L. Knapp of the Naval Research Laboratory, Washington, who obtained $207 \mathrm{~K}$ at $9.55 \mathrm{~mm}$ wavelength (ibid., 204) from which they conclude that the temperature is constant over a range from $1 \mathrm{~mm}$ to $21 \mathrm{~cm}$.

In a critical reassessment of the published data, including the forementioned results, E. Epstein concludes (ibid., 214) that the spectrum definitely does not turn up at short wavelengths as predicted by elementary theory. $\mathrm{He}$ has reduced to a common basis all measurements made with sensitive equipment. Furthermore, he has taken into account systematic errors caused by uncertainties in the flux densities of the primary calibrators and has rejected points with large errors. The final result of this weeding out process is a flat or slightly turned down spectrum, although the errors on individual points (typically $\pm 30 \mathrm{~K}$ ) are as large as the reduction at short wavelengths.
The final article, by Carl Sagan and Joseph Veverka (ibid., 222), is an objective examination of Epstein's conclusion that the simple theory must be incorrect. There are several ways of changing the argument by introducing internal sources of heat, ferromagnetic materials, differing electrical and thermal skin depths or radiative conduction, so that the heat budget of the planet is modified. None of these artificial devices will, however, reproduce Epstein's spectrum.

According to Sagan and Veverka the observed spectrum is obtained if there is a thin layer of material with high dielectric constant and high absorption coefficient close to the Martian surface. This layer must be entirely transparent to centimetre radiation, but strongly influence millimetre wave propagation. It is not very surprising to learn that a layer of liquid water on Mars would have just these properties. The total volume of liquid water needed-a layer $50 \mu$ thick-is not much larger than the amount already present in the tenuous atmosphere as vapour.

\title{
Membrane Bound Polyribosomes
}

WITHIN the cytoplasm of animal cells a fraction of the polyribosomes may be topologically segregated from the rest by attachment to membranes. This is particularly evident in tissues which secrete large amounts of protein, such as liver, pancreas and plasma cells, where ribosome-studded "rough endoplasmic reticulum" is most abundant. The immediate inference is that the ribosomes attached to the endoplasmic reticulum are largely those synthesizing proteins destined for streamlined package and export, and during the past few years data have been accumulating in support of this idea. For example, in liver, membrane bound polysomes synthesize predominantly serum proteins and free polysomes synthesize nonserum proteins such as ferretin (Ganoza and Williams, Proc. US Nat. Acad. Sci., 63. 1370; 1969; Hicks et al., Science, $164,584 ; 1969)$. An apparent prerequisite for the correct assembly of the light and heavy chains of immunoglobulin in lymph node cells is their discharge from membrane bound polysomes into the endoplasmic reticulum (Vassalli et al., J. Mol. Biol., 56, 1; 1971).

Although this evidence confirms preconceived ideas, it does raise some important questions about the mechanism of assembly and selective attachment of these polysomes to the endoplasmic reticulum. A report by Baglioni, Bleiberg and Zauderer in next Wednesday's Nature New Biology is the vanguard of what promises to be an all-out attack on these problems. Working with mouse myeloma cells which secrete a single immunoglobulin and have a well developed endoplasmic reticulum, Baglioni et al. consider three models for the assembly of membrane bound polysomes. These models incorporate the fact that polysomes are attached to the endoplasmic reticulum through their $60 \mathrm{~S}$ subunits, from which the nascent polypeptide chains probably extend towards the lumen, and the generally accepted idea that ribosomes join messenger RNA in the sequence $40 \mathrm{~S}$ ribosomal subunit plus "initiation factors", followed by the $60 \mathrm{~S}$ subunit.

In the first model ribosomes translating mRNA coding for an exported protein become attached to membranes only when the nascent polypeptide chain is long enough to recognize specific binding sites. Conversely, the mRNA-80S initiation complex may bind to the membrane immediately because of some specificity in the $60 \mathrm{~S}$ subunit, or an mRNA-40S complex may seek out a $60 \mathrm{~S}$ subunit already membrane bound.

Baglioni et al. distinguish between these alternatives by using a double isotope labelling technique to observe the entry of newly synthesized ribosomal subunits into the membrane bound and free ribosome populations. By carefully eliminating contamination of the first fraction by mitochondrial ribosomes and subunits they show that new $60 \mathrm{~S}$ subunits become attached to membrane independently of $40 \mathrm{~S}$ subunits and in the absence of protein synthesis, thus eliminating models one and two. 